belonging to the Company were at work during most of the year in the Atlantic and Pacific Oceans, the Mediterranean Sea, and the Persian Gulf. The magnetic detection of nuclear weapon effects, the simulation of lunar environments, the analysis of radar terrain, and oceanographic systems and services were other fields of investigation undertaken by the Company.

To satisfy the growing need for its products, Texas Instruments has embarked on an extensive building pro- gramme. A building for plating operations on the Central Expressway site and the doubling to 60,000 sq. ft. of the main plant of the Company's subsidiary, Engineering Supply Co., have been completed, and in November 1962 the construction began of a 60,000 sq. ft. components plant near Nice, France, similar to the plants in Dallas and England. This was followed, in December, by the start of a 290,000 sq. ft. multi-purpose manufacturing plant on the Central Expressway site.

\title{
RADIATION QUANTITIES AND UNITS
}

\begin{abstract}
HE reports of the International Commission on Radiological Units and Measurements have been published in past years by the National Bureau of Standards in the Handbook series, and each of the triennial reports has constituted a complete restatement of the recommendations of the Commission. During 1962 , however, the Commission decided to modify its previous practice and Report $10 a *$ is the first of a new series of reports, each of which deals with one aspect only of the work of the Commission.

Report $10 a$ deals with radiation quantities and units and presents the recommendations of the Commission agreed at its meeting held in Montreux, during April $2-14,1962$. Report $10 b$ will deal with physical aspects of irradiation, Report $10 \mathrm{c}$ with radioactivity, Report $10 d$ with clinical dosimetry, Report $10 e$ with radiobiological

* United States Department of Commerce: National Bureau of Standards. Handbook No. 84: Radiation Quantities and Units-International Com

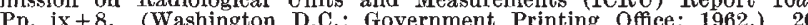
cents.
\end{abstract}

dosimetry, and Report $10 f$ with methods of evaluating radiological equipment.

The Commission recommends that the use of each special unit be restricted to one quantity, namely the radsolely for absorbed dose; the roentgen-for exposure; and the curie-for activity. Several new names are proposed. For protection purposes, the 'dose equivalent' $(D E)$ is introduced, defined as the product of absorbed dose, $D$, quality factor $(Q F)$, dose distribution factor $(D F)$ and other necessary modifying factors, and the unit of dose quivalent is the 'rem', being numerically equal to the dose in rads multiplied by the appropriate modifying factors. Another new name, "kerma", based on the initials of kinetic energy released in material, is for the quantity which represents the kinetic energy transferred to charged particles by the uncharged particles per unit mass of the irradiated medium. Others are 'energy fluence' with its related quantity 'particle fluence', and 'specific gamma ray constant'. The term 'activity' is recommended to be used for the transformation rate and its unit, the curie, defined as $3.7 \times 10^{10} \mathrm{sec}^{-1}$.

\section{THE UNSOLVED PROBLEM OF BALL LIGHTNING}

\section{BY STANLEY SINGER}

\author{
Rocket Power Inc., Research Laboratories, Pasadena, California
}

$\mathrm{T}$ HE International Conference on Atmospheric and Space Electricity, held on May 6-10 in Montreux, dealt with present problems of meteorological electrical phenomena. Scientists from twenty-five countries participated in discussions organized about major problem areas. Several of the most difficult questions were also those of the longest standing.

Storms in Nature exhibit marked electrical activity, the most readily observed result being ordinary lightning. One of the most controversial questions concerns the separation of charge in these storms. The processes which build up the electrical potentials and current involved in lightning have not been established. Laboratory investigations have provided information on charge separation occurring, for example, between the solid and liquid phases in the freezing of water ${ }^{1}$. The complexity and scale of natural storms have called forth more complex theories for which similar direct experimental data is lacking ${ }^{2}$. The problem of ball lightning has received much attention, including numerous experimental investigations; yet the available information is even more limited. The experiments have failed to duplicate major properties of the natural phenomenon, and it can only be said that promising explanations have been proposed.

Atmospheric potentials are responsible for several lightemitting phenomena, for example, the glowing spheres known as St. Elmo's fire. Although traditionally seen on the masts of sailing ships, these are also observed on trees, stones, and even human beings on mountain tops. St. Elmo's fire is usually attributed to the corona discharge caused by a potential at the tip of a protruding object such as a stake in the ground. The properties of this discharge account for the stable position of St. Elmo's fire. Ball lightning, on the contrary, can move over an extended path, according to many reports.

This extensive motion and other unusual properties have attracted notice to ball lightning although it is seen rarely compared with ordinary lightning. The great variety of phenomena reported during thunderstorms (including pinched lightning ${ }^{3}$, bead lightning, and St. Elmo's fire) and the nature of the reports have led to a not unreasonable suspicion of many observations of ball lightning. Witnesses are present by chance, usually in unsettling circumstances. Several accounts resemble folk-lore. It is not surprising, therefore, that some authorities, notably Humphreys ${ }^{4}$ and Schonland ${ }^{5}$, have expressed scepticism that ball lightning occurs. Humphreys examined 280 reported observations, and concluded that all but few were optical illusions resulting from an intense light such as ordinary lightning. The remaining few (less than half a dozen) he conceded might be examples of St. Elmo's fire. Several scientists, however, have reported viewing ball lightning; and photographs have been obtained ${ }^{6-8}$. None of the pictures is equal in quality to those available of the ordinary strokes of lightning, but two general aspects of the ball are recorded. One shows a stationary bright object not particularly spherical in shape, sometimes with glowing tentacles. The other records an illuminated path with well-defined boundaries apparently caused by motion of the ball. In view of continuing evidently authentic reports it appears that the best conclusion is that ball lightning does indeed exist. 